\title{
EFEITO DA RESTRIÇÃO ALIMENTAR SOBRE ALGUMAS CARACTERÍSTICAS DE CARCAÇA DE CABRITOS F1 BOER X SAANEN ${ }^{1}$
}

\author{
Effect of feed restriction on some carcass traits of F1-Boer $X$ Saanen goats
}

\author{
José Morais Pereira Filho ${ }^{2}$, Kleber Tomas de Resende ${ }^{3}$, Izabelle A. Molina de Almeida Teixeira ${ }^{4}$, \\ Américo Gárcia da Silva Sobrinho ${ }^{5}$, Enrique Alejandro Yáñez ${ }^{6}$, Angela Cristina Dias Ferreira ${ }^{7}$
}

\begin{abstract}
RESUMO
Objetivou-se com este trabalho avaliar o efeito da restrição alimentar nas características de carcaça de cabritos F1 Boer x Saanen. Foram utilizados 21 cabritos, pesando $15 \mathrm{~kg}$ de PV, distribuídos em três tratamentos ( 0,30 e $60 \%$ de restrição). O consumo dos animais do tratamento $0 \%$ de restrição determinavam o consumo dos animais dos tratamentos 30 e $60 \%$ de restrição. Quando os animais do nível de restrição $0 \%$ atingiam $25 \mathrm{~kg}$, estes juntamente com seus pares foram submetidos a jejum de sólido de $24 \mathrm{~h}$ e de líquido de $16 \mathrm{~h}$. O abate ocorreu mediante descarga elétrica, seguido de sangria e retirada dos órgãos. Os ganhos de peso foram de 211,03, 126,15 e 11,71g/dia; a eficiência alimentar de 0,20, 0,18 e de 0,03; os pesos de abate de 25,44,20,91 e 15,82kg para os tratamentos 0,30 e $60 \%$ de restrição, respectivamente. $\mathrm{O}$ rendimento de carcaça quente, de carcaça fria e biológico não foram influenciados pela restrição alimentar. Somente a proporção da paleta e a do lombo foram influenciados pela restrição alimentar, com aumento linear do rendimento da paleta e decréscimo linear do rendimento do lombo. Houve efeito da restrição na redução do rendimento de gordura e aumento da proporção de osso. A restrição alimentar em níveis moderados, permitiu a obtenção de carcaças de boa qualidade, com bom rendimento, elevada proporção de músculo e baixa participação de gordura e, dependendo da relação custo:benefício, pode tornar-se boa alternativa para o produtor.
\end{abstract}

Termos para indexação: Paleta, perna, peso de corpo vazio, rendimento de carcaça fria.

\section{ABSTRACT}

To evaluate the effect of different levels of feed restriction on carcass traits, twenty-one F1-Boer $\mathrm{x}$ Saanen goats weighting 15 $\mathrm{kg} \mathrm{LW}$ were allocated to one of the three treatments $(0,30$ and $60 \%$ restriction). The feed intake for the $0 \%$ restriction treatment animals determined the intake for the animals in the 30 and $60 \%$ restriction treatment. When animals from $0 \%$ of feed restriction reached $25 \mathrm{~kg}$ of LW, they and their pair-feeding were submitted to a feed and water fasting during $24 \mathrm{~h}$ and $16 \mathrm{~h}$, respectively. After fasting, animals were slaughtered by electric discharged. In addition, bleeding and organs removing were performed. The weight gain (g/day) were $211,03,126,15$ and 11,71 ; the feed efficiency was $0,20,0,18$ and 0,03 ; the slaughtering weight $(\mathrm{kg}$ ) were $25,44,20,91$ and 15,82 for 0,30 e $60 \%$ restriction treatment, respectively. Warn carcass, cold carcass and biological yields were no affected by feed restriction. Only, shoulder and loin proportions were affected by feed restriction with a linear increase of shoulder yield and a linear decrease of loin yield. Feed restriction reduced fat yield and increased bone proportion. Moderate levels of feed restriction permitted to produce goat carcasses with good quality and yield, as well as high muscle proportion and low fat content. Depends on the cost: benefit ratio feed restriction should be consider an adequate alternative for meat goat producer.

Index terms: Pallete, leg, empty body weight, cold carcass yield.

\section{(Recebido em 3 de Janeiro de 2005 e aprovado em 13 de julho de 2006)}

\section{INTRODUÇÃO}

Nos últimos anos, a criação de caprinos no Brasil vem estabelecendo-se como atividade econômica, especialmente para o pequeno e médio criador, que através da produção de leite e/ou carne, bem como de seus derivados, vem ocupando nichos de mercado que demandam produtos de melhor qualidade. Por outro lado, a exploração de caprinos leiteiros na fase de aleitamento é caracterizada como onerosa, de pouco ou nenhum retorno econômico, fato que tem levado ao sacrifício os cabritos logo após o nascimento (RESENDE, 2002).

A integração leite-carne tem despertado interesse de produtores, principalmente nos estados da região Sudeste, o que tem ocasionado cruzamentos de reprodutores Boer com parte das fêmeas leiteiras, objetivando o abate de machos e fêmeas de forma a agregar valor ao sistema de produção. Neste caso, a criação de caprinos para a comercialização ao final do aleitamento, com carcaças pesando de 5 a $6 \mathrm{~kg}$, ou o abate de animais

\footnotetext{
Parte da tese do primeiro autor apresentada à Faculdade de Ciências Agrárias e Veterinárias da UNESP/Jaboticabal, SP.

${ }^{2}$ Médico Veterinário, Doutor doDepartamento de Medicina Veterinária da Universidade Federal de Campina Grande - Cx. P. 64 - 58708-110 Patos, PB - jmorais@cstr.ufcg.edu.br

${ }^{3}$ Zootecnista, Doutor do Departamento de Zootecnia - Faculdade de Ciências Agrárias e Veterinárias/FCAV/UNESP - Via de acesso Paulo Donato Castellane s/n - 14.884-900 - Jaboticabal, SP - kresende@fcav.unesp.br

${ }^{4}$ Zootecnista, Doutora do Departamento de Zootecnia - FCAV/UNESP - Via de acesso Paulo Donato Castellane s/n - 14.884-900 - Jaboticabal, SP _ izabelle@fcav.unesp.br

${ }^{5}$ Zootecnista, Doutor do Departamento de Zootecnia - FCAV/UNESP - Via de acesso Paulo Donato Castellane s/n - 14.884-900 - Jaboticabal, SP _ americo@fcav.unesp.br

${ }^{6}$ Médico Veterinário, Doutor do Departamento de Procucción Animal Facultad de Ciencias Veterinarias da UNNE - Rua Sargento Cabral 2139 Corrientes - Argentina, 3400 - eyanez@vet.unne.edu.ar

${ }^{7}$ Zootecnista, Doutora, Professora do Departamento de Zootecnia da Unoeste - Rua José Bongiovani, 700 - 19.050-900 - Presidente Prudente, SP _ acrisdias@hotmail.com
} 
terminados em confinamento com 25 a $35 \mathrm{~kg}$ de PV e carcaça de 12 a $15 \mathrm{~kg}$ pode tornar-se alternativa viável (PEREIRA FILHO et al., 2005).

Os custos de produção e a ausência de padronização de carcaça e de cortes comerciais caracterizam-se como pontos críticos para produção de carne caprina. Para Ribeiro (1997), na caprinocultura leiteira, a alimentação é responsável por 50 a $60 \%$ dos custos de produção, podendo representar até $80 \%$. Entre as alternativas usadas para reduzir custos na alimentação de ruminantes destaca-se: substituição de concentrado por volumoso (MORON-FUENMAYOR \& CLAVERO, 1999); uso de níveis crescentes de proteína (MTENGA \& KITALY, 1990); restrição alimentar temporária e exploração do crescimento compensatório (SAHLU et al., 1999); restrição quantitativa, com dieta única e níveis crescentes de restrição (YÁÑEZ, 2002). Porém, no Brasil, a formulação de rações para caprinos seguem tabelas de comitês internacionais, que, segundo Resende et al. (2001), não predizem eficientemente a resposta animal, dificultando ainda mais qualquer programa de restrição alimentar.

Revisão feita por Warmington \& Kirton (1990) demonstrou que a manipulação nutricional em caprinos pode influenciar a distribuição de gordura corporal. Yáñez (2002) avaliou os efeitos dos níveis 0\%, 30\% e 60\% de restrição e observou que os rendimentos de carcaça e de cortes dos animais com $0 \%$ e $30 \%$ de restrição foram semelhantes, o que levou o autor a sugerir que restrição alimentar moderada pode reduzir os custos de produção sem comprometer a qualidade da carcaça. Para Warmington \& Kirton (1990), o peso da carcaça é função da taxa de crescimento e do rendimento de carcaça que, por sua vez, varia segundo o grupo genético, o sexo, a idade, a condição fisiológica e a nutrição. Já a qualidade da carcaça é determinada pela distribuição de músculo, osso e gordura.

Yáñez (2002) observou efeito linear decrescente da restrição alimentar sobre o rendimento de carcaça fria, rendimento de lombo e proporção de gordura na perna de cabritos Saanen. Pralomkarn et al. (1995) não observaram efeito da restrição sobre a proporção de músculo, osso e gordura na carcaça ao avaliar o efeito dos níveis de mantença, 1,2 e 1,9 vezes a mantença em caprinos Thai nativo, Aglo-nubiano e mestiços Thai x Aglo-nubiano. Nesse sentido, a restrição alimentar como prática de manejo nutricional pode refletir positivamente nas características da carcaça dos caprinos. Portanto, o objetivo deste trabalho foi avaliar o efeito da restrição alimentar sobre as características de carcaça e dos cortes comerciais de cabritos F1 Boer x Saanen.

\section{MATERIAL E MÉTODOS}

O trabalho foi realizado no Setor de Caprinos da FCAV/Unesp, em Jaboticabal, SP. Os animais foram alojados em gaiolas medindo $0,50 \times 1,00 \mathrm{~m}$, com bebedouro e comedouro individuais. Foram utilizados 21 cabritos inteiros, F1 Boer x Saanen, pesando $15 \mathrm{~kg}$ de peso vivo (PV), os quais foram submetidos a três níveis de restrição alimentar (0\% - alimentação à vontade, 30 e $60 \%$ de restrição em relação ao ingerido pelos animais alimentados à vontade) de uma dieta formulada com base nas exigências em proteína e energia metabolizável para um ganho de 150 gramas/dia (AFRC, 1998).

$A$ ração foi fornecida às $7 \mathrm{~h}$, com ajustes diários para permitir sobra de $20 \%$ aos animais do tratamento com $0 \%$ de restrição. Os ingredientes utilizados na ração foram: feno da planta de milho (46,88\%), milho moído $(25,91 \%)$, farelo de soja $(19,32 \%)$, melaço de cana-de-acúcar $(4,29 \%)$, óleo de soja $(0,81 \%)$, núcleo mineral $(1,99 \%)$ e calcário calcítico $(0,80 \%)$. A composição químico-bromatológica da dieta é apresentada na Tabela 1.

As análises de MS, PB, EE, FDN, FDA, cálcio e fósforo foram realizadas no Laboratório de Nutrição Animal da FCAV/Unesp, segundo a metodologia descrita por Silva \& Queiroz (2002). A energia metabolizável foi obtida a partir do ensaio de digestibilidade e da estimativa da produção de gases no rúmen (BLAXTER, 1962).

Foram realizadas pesagem a cada semana até os animais do nível de restrição $0 \%$ atingir $25 \mathrm{~kg}$ de PV, quando, juntamente com seus pares, foram submetidos a jejum de sólido de 24 horas e de líquido de 16 horas. Os animais foram pesados antes e após o jejum, obtendo-se o peso vivo (PV) e o peso em jejum (PJ). O abate foi feito mediante insensibilização elétrica, seguido de sangria e retirada dos órgãos. $\mathrm{O}$ trato gastrintestinal foi esvaziado e limpo para a obtenção do peso do corpo vazio (PCV), que foi calculado subtraindo o conteúdo gastrintestinal e o líquido contido na bexiga e na vesícula biliar do PJ. A carcaça foi obtida após a separação das mãos e dos pés na articulação carpo metacarpiana e tarso metatarsiana, respectivamente, obtendo-se o peso da carcaça quente (PCQ). As carcaças foram mantidas em câmara fria por 24 horas, em temperatura de $5^{\circ} \mathrm{C}$, obtendo-se o peso da carcaça fria (PCF). A estimativa do rendimento de carcaça quente (RCQ), rendimento de carcaça fria $(\mathrm{RCF})$ e rendimento biológico (RB) foi feita de acordo com a metodologia descrita por Osório et al. (1998). 
TABELA 1 - Composição químico-bromatológica da dieta.

\begin{tabular}{|c|c|}
\hline Componente & $\%$ \\
\hline Matéria seca & 89,32 \\
\hline Proteína bruta ${ }^{1}$ & 16,50 \\
\hline Extrato etéreo $^{1}$ & 3,56 \\
\hline Energia metabolizável (Mcal/kg MS) & $2,46^{*}$ \\
\hline Fibra em detergente neutro ${ }^{1}$ & 48,82 \\
\hline Fibra em detergente ácido ${ }^{1}$ & 17,72 \\
\hline Cálcio ${ }^{1}$ & 0,65 \\
\hline Fósforo ${ }^{1}$ & 0,32 \\
\hline
\end{tabular}

${ }^{1}$ valores expressos com base na matéria seca.

* = obtido a partir da digestibilidade e da estimativa da produção de gases (BLAXTER, 1962).

A carcaça foi seccionada e a meia-carcaça esquerda foi dividida em cinco cortes: perna, lombo, costelas, paleta e pescoço, os quais foram obtidos segundo adaptações das metodologias de Colomer-Rocher et al. (1987), Garcia (1998), Osório et al. (1998) e Yáñez (2002). A base óssea e a região de secção dos cortes foram: perna, que abrange a região do ilíaco (ílio), ísquio, púbis, vértebras sacrais, as duas primeiras vértebras coccígeas, fêmur, tíbia e tarso; lombo, compreendendo toda a região das vértebras lombares; costelas, que incluem o esterno e todas as costelas e vértebras torácicas; paleta, correspondendo à região da escápula, úmero, rádio, ulna e o carpo; pescoço, região correspondente as sete vértebras cervicais.

A perna de cada animal foi congelada a $-20^{\circ} \mathrm{C}$ para, posteriormente, ser descongelada e dissecada em músculo, osso, gordura e outros tecidos. Os resultados foram expressos em peso absoluto e em relação ao peso da perna. A dissecação foi feita segundo a metodologia descrita por Yáñez (2002).

$\mathrm{O}$ delineamento experimental foi o inteiramente casualizado, com três tratamentos e sete repetições. Os dados foram avaliados através da análise de variância e de regressão, segundo os procedimentos Proc Glm e Proc Reg do SAS Institute (1999).

\section{RESULTADOS E DISCUSSÃO}

Embora os resultados de consumo e de desempenho dos animais não tenham sido avaliados neste trabalho, é importante destacar que a oferecimento de matéria seca dos animais do tratamento $0 \%$ de restrição foi ajustada diariamente para permitir sobras de $20 \%$, e a partir do ingerido por estes animais aplicava-se a restrição de 30 e $60 \%$, o que possibilitou durante o experimento ingestão total de 47,67, 32,74 e 19,45 kg de MS; e em relação ao peso vivo (PV) de 4,47, 3,66 e $2,87 \%$ do PV para os tratamentos 0,30 e $60 \%$ de restrição, respectivamente. Já em termos de desempenho os resultados foram os seguintes: $211,03,126,15$ e $11,71 \mathrm{~g} / \mathrm{dia}$ de ganho de peso; 0,20, 0,18 e de 0,03 de eficiência alimentar; $25,44,20,91$ e $15,82 \mathrm{~kg}$ de pesos ao abate; $112,71,113,57 \mathrm{e}$ 112,57 dias de idades ao abate, respectivamente para 0,30 e $60 \%$ de restrição. De acordo com Pereira Filho et al. (2005), estes resultados indicam que a restrição alimentar praticada em níveis moderados mantém a eficiência alimentar e reduz as perdas com alimentação e diminui, gradativamente, os custos com alimentação.

Houve efeito linear negativo $(\mathrm{p}<0,05)$ da restrição para PV, PJ, PCV, PCQ, PCF e PR, mas os índices de rendimento não foram influenciados pela redução na oferta de alimento (TABELA 2). Como os animais foram alimentados à vontade até $15 \mathrm{~kg}$ de $\mathrm{PV}$ e apresentavam no início do experimento idade média de 67 dias e já haviam sido desaleitados (50 dias), é possível que os pré-estômagos estivessem próximo de completar seu desenvolvimento, reduzindo o efeito da restrição sobre o desenvolvimento do animal.

Pralomkarn et al. (1995), avaliando o efeito do genótipo (Thai nativo e Thai nativo x Anglo-nubiano) e da restrição alimentar (mantença, 1,2 e 1,9 vezes a mantença), obtiveram, ao final de 7 semanas, efeito linear positivo para PCV; entretanto, não foi observado efeito sobre o rendimento biológico, que variou de $54,2 \%$ a $55,5 \%$, resultados próximos dos $56,35 \%, 57,78 \%$ e $57,04 \%$ obtidos neste trabalho com os animais submetidos a $0 \%, 30 \%$ e $60 \%$ de restrição, respectivamente. Resultados que podem ser atribuído à quantidade e proporção do conteúdo gastrintestinal em relação ao PCV que foram de $3892,9 \mathrm{~g} \mathrm{e} 16,4 \%, 3462,8 \mathrm{~g}$ e $17,4 \%, 2957,5$ g e 19,6\% nos animais submetidos a $0 \%, 30$ e $60 \%$ de restrição, respectivamente; podendo ainda ser relacionado ao aumento da gordura na carcaça dos animais alimentados à vontade, cujo peso de abate foi de $25,4 \mathrm{~kg}$ contra os $15,8 \mathrm{~kg}$ dos animais com $60 \%$ de restrição. 
TABELA 2 - Médias e equações de regressão para peso vivo (PV), peso em jejum (PJ), peso de corpo vazio (PCV), peso de carcaça quente (PCQ), peso de carcaça fria (PCF), rendimentos de carcaça quente (RCQ) e de carcaça fria (RCF), rendimento biológico $(\mathrm{RB})$ e perda pelo resfriamento $(\mathrm{PR})$ da carcaça de cabritos F1 Boer x Saanen, em função da restrição alimentar.

\begin{tabular}{lrrrrrrr}
\hline \multirow{2}{*}{ Item } & \multicolumn{3}{c}{ Restrição } & & Equações & $\mathbf{R}^{2}$ & P \\
\cline { 2 - 4 } & $\mathbf{0 \%}$ & $\mathbf{3 0 \%}$ & $\mathbf{6 0 \%}$ & & & \\
\hline PV (kg) & 25,44 & 21,17 & 15,77 & & $\hat{Y}=25,587-0,161 X$ & 0,95 & 0,0001 \\
PJ (kg) & 23,68 & 19,91 & 15,06 & & $\hat{Y}=23,810-0,147 X$ & 0,96 & 0,0001 \\
PCV (kg) & 19,79 & 16,44 & 12,10 & & $\hat{Y}=19,935-0,129 X$ & 0,95 & 0,0001 \\
PCQ (kg) & 11,15 & 9,51 & 6,91 & & $\hat{Y}=11,283-0,072 X$ & 0,89 & 0,0001 \\
PCF (kg) & 10,82 & 9,19 & 6,61 & & $\hat{Y}=10,946-0,071 X$ & 0,89 & 0,0001 \\
RCQ (\%) & 47,11 & 47,73 & 45,85 & & $\hat{Y}=47,489$ & 0,06 & 0,3150 \\
RCF (\%) & 45,70 & 46,11 & 43,87 & & $\hat{Y}=45,275$ & 0,11 & 0,1818 \\
RB (\%) & 56,35 & 57,78 & 57,04 & $\hat{Y}=56,826$ & 0,01 & 0,7710 \\
PR (\%) & 3,11 & 3,52 & 4,51 & $\hat{Y}=3,013+0,023 X$ & 0,54 & 0,0097 \\
\hline
\end{tabular}

$\hat{\mathrm{Y}}=$ variável dependente; $\mathrm{X}=$ nível de restrição.

$\mathrm{R}^{2}=$ coeficientes de determinação; $\mathrm{P}=$ probabilidade.

Para Warmington \& Kirton (1990), a influência da nutrição sobre o rendimento de carcaça está associada principalmente a variações no peso do conteúdo gastrintestinal e dos órgãos internos, ressaltando ainda que a restrição quantitativa e o aumento do teor de fibra da dieta proporcionam maior tempo de retenção do alimento no trato digestório, resultando em menor rendimento de carcaça.

O efeito linear positivo $(\mathrm{p}<0,05)$ da restrição sobre a PR pode estar relacionado ao atendimento das exigências nutricionais de manutenção dos animais com $60 \%$ de restrição, enquanto os animais com $0 \%$ de restrição, se desenvolveram de forma adequada, com melhor desempenho diário e maior peso de abate, que segundo Yáñez (2002) tem relação direta com a composição corporal, ou seja, proporção elevadas de proteína possibilita aumento na perda de água. Por outro lado, a maior deposição de gordura corporal, inclusive da gordura subcutânea, resulta em proteção da carcaça durante o resfriamento, diminuindo a PR, que neste trabalho foi de $4,51 \%$ nos animais com $60 \%$ de restrição e de apenas $3,11 \%$ nos animais alimentados à vontade.

O peso dos cortes comerciais e a proporção dos cortes na carcaça são descritos na Tabela 3 . O peso de todos os cortes diminuiu linearmente $(\mathrm{p}<0,05)$ à medida que foi aumentando a restrição alimentar, refletindo a diferença verificada no PV desses animais. Os resultados referentes à paleta, ao pescoço, ao lombo e à perna foram semelhantes aos obtidos por Yáñez (2002) para cabritos da raça Saanen de 20 a $35 \mathrm{~kg}$ de PV. A idade de abate variou de 94 a 127 dias e o peso ao nascer variou de 3,2 a $5,1 \mathrm{~kg}$, e ambos foram testados como co-variáveis e não foram significativos.

Somente a paleta e o lombo foram influenciados pela restrição alimentar, com aumento linear $(\mathrm{p}<0,05)$ de 0,031 e de 0,033 na proporção da paleta e do lombo na carcaça, para cada unidade percentual restrita na dieta. Yáñez (2002), trabalhando com a mesma alimentação e os mesmos níveis de restrição, obteve aumento linear no rendimento da paleta e do pescoço, e quanto à participação do lombo, o autor observou decréscimo linear, diferentemente do que foi obtido neste trabalho.

São poucos os trabalhos que avaliam a proporção e a relação entre os tecidos da carcaça de caprinos. Alguns trabalhos utilizam cortes para estimar a composição da carcaça, mas Warmington \& Kirton (1990) ressaltam que a ausência de padronização de cortes comerciais e as diferentes adaptações metodológicas de dissecação dificultam a exploração mais detalhada dos resultados. Mesmo assim, os autores destacam que, em animais confinados, a proporção de músculo pode variar de 60 a $70 \%$, a de osso entre 12 a $29 \%$ e a de gordura oscilando de 5 a $24 \%$, enquanto em animais criados em pastejo as proporções variam de 56 a $68 \%$ de músculo, 16 a $44 \%$ de osso e 4 a $32 \%$ de gordura. 
O peso de todos os tecidos decresceu linearmente ( $p<0,05)$ com o aumento do nível de restrição alimentar (Tabela 4). A grande variabilidade no peso da gordura e de outros tecidos, em parte, pode ser associada à aplicação da metodologia de dissecação, que dificulta a distinção desses componentes. Com relação à proporção dos tecidos na perna, ocorreu efeito da restrição somente na redução do rendimento de gordura e no aumento da proporção de osso (Tabela 3), resultados semelhantes foram observados por Yáñez (2002) para cabritos Saanen abatidos com PV entre 20 e $35 \mathrm{~kg}$. Oman et al. (1999) obtiveram 62,52\% de músculo, $31,01 \%$ de osso e $6,74 \%$ de gordura na perna de caprinos Spanish pesando 33,52 kg de PV e para os mestiços (Boer $\mathrm{x}$ Spanish) com 38,17 kg de PV verificaram para músculo, osso e gordura proporções de 62,23\%, 29,54\% e 8,23\%, respectivamente. Estes valores podem ser considerados inferiores aos $68,38 \%$ de músculo, superiores aos $17,42 \%$ de osso e próximos aos $9,63 \%$ de gordura obtida com os animais mantidos com $0 \%$ de restrição.

A relação M:O diminuiu linearmente $(\mathrm{p}<0,05)$, enquanto as relações músculo:gordura (M:G) e osso:gordura $(\mathrm{O}: \mathrm{G})$ aumentaram à medida que se intensificou a restrição (Tabela 4), comportamento que reflete os níveis de ingestão em cada tratamento, pois o direcionamento dos nutrientes determinado pelo o organismo atende, prioritariamente, aos tecidos nervoso e ósseo, seguido do muscular e adiposo. Estes resultados indicam que, dependendo do objetivo, a restrição pode ser recomendada até o nível que garanta a melhor resposta econômica ao produtor sem afetar a qualidade da carcaça exigida pelo consumidor.

Diferentemente dos resultados obtidos neste trabalho, Pralomkarn et al. (1995) não observaram influência da restrição sobre as relações músculo:osso e músculo + gordura:osso. A relação M:O foi semelhante à obtida com caprinos Flórida nativo (JOHNSON \& MCGOWAN, 1998) e a observada em mestiços Aglo-nubiano x Flórida nativo e Spanish x Flórida nativo por Johnson et al. (1995). Todavia, a relação M:O foi superior à obtida por Srivastava \& Sharma (1997) para animais Jamnapari.

Os animais apresentaram menor deposição de gordura na carcaça, quando comparados com os cabritos Saanen de 20 a $35 \mathrm{~kg}$ avaliados por Yáñez (2002), que apresentaram maior deposição de gordura, sobretudo de cobertura, fato que o autor relacionou com a castração dos animais, diferentemente dos animais estudados neste trabalho, que não foram castrados.

Além das variáveis estudadas neste trabalho, verificase a necessidade de incorporação de variáveis associadas à preferência dos consumidores e a viabilidade econômica para o produtor. Portanto, o estudo das características de carcaça em caprinos, sobretudo a avaliação de desenvolvimento alométrico dos tecidos, composição química e características organolépticas da carne e da gordura precisam continuar, e, necessariamente, devem evoluir no sentido de atender às exigências do mercado.

TABELA 3 - Médias e equações de regressão para o peso dos cortes comerciais e a proporção dos cortes na carcaça de cabritos F1 Boer x Saanen, em função da restrição alimentar.

\begin{tabular}{lrrrlcrr}
\hline \multirow{2}{*}{ Corte } & \multicolumn{3}{c}{ Restrição } & & Equações & \multirow{2}{*}{$\mathbf{R}^{\mathbf{2}}$} & P \\
\cline { 2 - 4 } & $\mathbf{0 \%}$ & $\mathbf{3 0 \%}$ & $\mathbf{6 0 \%}$ & & & \\
\hline Perna (g) & 1715,71 & 1455,71 & 1064,28 & $\hat{\mathrm{Y}}=1737,619-10,857 \mathrm{X}$ & 0,88 & 0,0001 \\
Lombo (g) & 704,28 & 538,57 & 365,71 & $\hat{\mathrm{Y}}=705,476-5,643 \mathrm{X}$ & 0,93 & 0,0001 \\
Costelas (g) & 1481,43 & 1262,86 & 910,00 & $\hat{\mathrm{Y}}=1503,809-9,524 \mathrm{X}$ & 0,87 & 0,0001 \\
Paleta (g) & 1035,71 & 921,43 & 697,14 & $\hat{\mathrm{Y}}=1054,048-5,643 \mathrm{X}$ & 0,77 & 0,0001 \\
Pescoço (g) & 538,57 & 484,28 & 317,14 & $\hat{\mathrm{Y}}=557,381-3,690 \mathrm{X}$ & 0,61 & 0,0001 \\
Perna (\%) & 31,34 & 31,20 & 31,72 & $\hat{\mathrm{Y}}=31,424$ & 0,03 & 0,1200 \\
Lombo (\%) & 12,87 & 11,57 & 10,90 & $\hat{\mathrm{Y}}=12,767-0,033 \mathrm{X}$ & 0,58 & 0,0006 \\
Costelas (\%) & 27,04 & 27,11 & 27,10 & $\hat{\mathrm{Y}}=27,084$ & 0,01 & 0,2245 \\
Paleta (\%) & 18,92 & 19,72 & 20,80 & $\hat{\mathrm{Y}}=18,872+0,031 \mathrm{X}$ & 0,48 & 0,0005 \\
Pescoço (\%) & 9,83 & 10,39 & 9,47 & $\hat{\mathrm{Y}}=9,898$ & 0,01 & 0,5879 \\
\hline
\end{tabular}

$\hat{\mathrm{Y}}=$ variável dependente; $\mathrm{X}=$ nível de restrição.

$\mathrm{R}^{2}=$ coeficientes de determinação; $\mathrm{P}=$ probabilidade. 
TABELA 4 - Médias e equações de regressão para peso, proporção e relação entre os componentes músculo (M), gordura $(\mathrm{G})$, osso $(\mathrm{O})$ e outros tecidos $(\mathrm{OT})$ da perna de cabritos F1 Boer x Saanen, em função da restrição alimentar.

\begin{tabular}{|c|c|c|c|c|c|c|}
\hline \multirow[b]{2}{*}{ Item } & \multicolumn{3}{|c|}{ Restrição } & \multirow[b]{2}{*}{ Equações } & \multirow[b]{2}{*}{$\mathbf{R}^{2}$} & \multirow[b]{2}{*}{$\mathbf{P}$} \\
\hline & $\begin{array}{c}0 \% \\
(1582,16)^{1} \\
\end{array}$ & $\begin{array}{c}30 \% \\
(1241,38)^{1}\end{array}$ & $\begin{array}{c}600 \% \\
(982,79)^{1} \\
\end{array}$ & & & \\
\hline Músculo (g) & 1083,80 & 932,08 & 676,74 & $\hat{\mathrm{Y}}=1100,642-6,741 \mathrm{X}$ & 0,79 & 0,0001 \\
\hline Osso (g) & 275,15 & 245,98 & 203,04 & $\hat{\mathrm{Y}}=277,587-1,216 \mathrm{X}$ & 0,76 & 0,0001 \\
\hline Gordura (g) & 152,38 & 108,34 & 56,22 & $\hat{Y}=153,688-1,599 X$ & 0,83 & 0,0001 \\
\hline OT (g) & 70,83 & 54,55 & 46,79 & $\hat{Y}=69,947-0,454 X$ & 0,50 & 0,0005 \\
\hline Músculo (\%) & 68,46 & 69,38 & 68,81 & $\hat{Y}=68,991$ & 0,03 & 0,5460 \\
\hline Osso (\%) & 17,42 & 18,41 & 20,70 & $\hat{Y}=17,213+0,054 X$ & 0,51 & 0,0003 \\
\hline Gordura (\%) & 9,63 & 8,13 & 5,71 & $\hat{Y}=9,778-0,065 X$ & 0,59 & 0,0001 \\
\hline OT (\%) & 4,46 & 4,08 & 4,76 & $\hat{\mathrm{Y}}=4,330$ & 0,0001 & 0,9953 \\
\hline $\mathrm{M}: \mathrm{O}(\mathrm{g} / \mathrm{g})$ & 3,96 & 3,79 & 3,33 & $\hat{Y}=4,004-0,010 X$ & 0,35 & 0,0087 \\
\hline $\mathrm{M}: \mathrm{G}(\mathrm{g} / \mathrm{g})$ & 7,22 & 8,87 & 12,25 & $\hat{Y}=6,945+0,083 X$ & 0,67 & 0,0001 \\
\hline $\mathrm{O}: \mathrm{G}(\mathrm{g} / \mathrm{g})$ & 1,83 & 2,36 & 3,68 & $\hat{Y}=1,706+0,030 X$ & 0,70 & 0,0001 \\
\hline
\end{tabular}

$\hat{Y}=$ variável dependente; $\mathrm{X}=$ nível de restrição; $\mathrm{R}^{2}$ = coeficientes de determinação;

$\mathrm{P}=$ probabilidade; ()$^{1}$ peso da perna obtido pela recomposição dos tecidos dissecados.

\section{CONCLUSÃO}

Se o objetivo for obter carcaças de boa qualidade e que apresentem bom rendimento comercial, elevada proporção de músculo e baixa participação de gordura e, dependendo da relação custo:benefício, a restrição alimentar moderada (até 30\%) pode tornar-se alternativa viável para o produtor.

\section{REFERÊNCIAS BIBLIOGRÁFICAS}

AGRICULTURAL AND FOOD RESEARCH COUNCIL. The nutrition of goat. Nutrition Abstract Revision (Series B), Aberdeen, v. 67, n. 11, p. 118, 1998.

BLAXTER, K. L. The energy metabolism of ruminants. London: Hutchinson \& Company, 1962. 329 p.

COLOMER-ROCHER, F.; MORAND-FEHR, P.; KIRTON, A. H. Standard methods and procedures for goat carcass evaluation, jointing and tissue separation. Livestock Production Science, Amsterdam, v. 17, p. 149-159, 1987.

GARCIA, C. A. Avaliação do resíduo de panificação "biscoito" na alimentação de ovinos e nas características quantitativas e qualitativas da carcaça. 1998. 78 f. Dissertação (Mestrado em Zootecnia) - Universidade Estadual Paulista, Jaboticabal, 1998.
JOHNSON, D. D.; McGOWAN, C. H. Diet/management effects on carcass attributes and meat quality of young goats. Small Ruminant Research, [S.1.], v. 28, p. 93-98, 1998.

JOHNSON, D. D.; McGAWAN, C. H.; NURSE, G. Breed type and sex effects on carcass traits, composition and tenderness of young goats. Small Ruminant Research, [S.1.], v. 17, p. 57-63, 1995.

MORON-FUENMAYOR, O. E.; CLAVERO, T. The effect of feeding system on carcass characteristics, non-carcass components and retail cut percentages of lambs. Small Ruminant Research, [S.1.], v. 34, p. 57-64, 1999.

MTEMGA, L. A.; KITALY, A. J. Growth perfornance and carcass characteristics of Tanzanian goats fed Chloris gayana hay with different levels of protein supplement. Small Ruminant Research, [S.1.], v. 3, p. 1-8, 1990.

OMAN, J. S.; WALDRON, D. F.; GRIFFIN, D. B. Effect of breed-type and feeding regimen on goat carcass traits. Journal Animal Science, Champaign, v. 77, p. 3215-3218, 1999.

OSÓRIO, J. C.; OSÓRIO, M. T.; JARDIM, P. O. Métodos para avaliação da produção da carne ovina: in vivo, na carcaça e na carne. Pelotas: UFPel, 1998. 107 p. 
PEREIRA FILHO, J. M.; RESENDE, K. T.; TEIXEIRA, I. A. M. A. Efeito da restrição alimentar no desempenho produtivo e econômico de cabritos F1 Boer x Saanen. Revista Brasileira de Zootecnia, Viçosa, v. 34, n. 1, p. 188196, 2005.

PRALOMKARN, W.; SAITHANOO, S.; KOCHAPAKDEE, $S$. Effect of genotype and plane of nutrition on carcass characteristics of Thai native and Anglo-nubian X Thai native male goats. Small Ruminant Research, [S.1.], v. 16, p. $21-25,1995$.

RESENDE, K. T. Distribuindo os partos ao longo do ano: o sistema da Unesp - Jaboticabal. Espírito Santo do Pinhal: Capritec, 2002. Disponível em: Liwww.capritec.com.bry. Acesso em: 8 out. 2002.

RESENDE, K. T.; PEREIRA FILHO, J. M.; TRINDADE, I. A. C. M. Exigências nutricionais de caprinos leiteiros. In:

Produção animal na visão dos brasileiros. Piracicaba: Sociedade Brasileira de Zootecnia, 2001. p. 484-496.

RIBEIRO, S. D. A. Caprinocultura: criação racional de caprinos. São Paulo: [s.n.], 1997. 311 p.
SAHLU, T.; HART, S. P.; GOETSCH, A. L. Effects of level of feed intake on body weight, body components, and mohair growth in Angora goats during realimentation. Small Ruminant Research, [S.1.], v. 32, p. 251-259, 1999.

SILVA, D. J.; QUEIROZ, A. C. Análise de alimentos: métodos químicos e biológicos. Viçosa: UFV, 2003. 237 p.

SRIVASTAVA, S. N. L.; SHARMA, K. Effect of feeding pelleted Leucaena leucocephala leaves on the carcass traits of Jamnapari goats. Indian Journal of Animal Sciences, New Delhi, v. 67, n. 2, p. 165-167, 1997.

SAS INSTITUTE. User's guide. North Caroline, 1999.

WARMINGTON, B. G.; KIRTON, A. H. Genetic and nongenetic influences on growth and carcass traits of goats. Small Ruminant Research, [S.1.], v. 3, p. 147-165, 1990.

YÁÑEZ, E. A. Desenvolvimento relativo dos tecidos e características da carcaça de cabritos Saanen, com diferentes pesos e níveis nutricionais. 2002. $85 \mathrm{f}$. Tese (Doutorado em Zootecnia) - Universidade Estadual Paulista, Jaboticabal, 2002. 